Abanico Veterinario. Enero-Diciembre 2021; 11:1-15. http://dx.doi.org/10.21929/abavet2021.30

Artículo Original. Recibido: 04/08/2020. Aceptado: 08/07/2021. Publicado: 28/07/2021. Clave: e2020-76.

\title{
Respuesta productiva y balance de nitrógeno en pollos adicionados con sustancias húmicas en el agua de bebida
}

\author{
Productive responses and nitrogen balance in broilers fed with humic substances in the \\ drinking water
}

\section{Sergio Gómez-Rosales ${ }^{1 *}$ ID, María Angeles ${ }^{1}$ ID, Jesús Maguey-González ${ }^{2}$ ID}

${ }^{1}$ Centro Nacional de Investigación Disciplinaria en Fisiología y Mejoramiento Animal, Instituto Nacional de Investigaciones Forestales Agrícolas y Pecuarias. Km. 1 Carretera a Colón, Ajuchitlán, Colón, Querétaro, México. CP 76280. 'Facultad de Estudios Superiores Cuautitlán, Universidad Nacional Autónoma de México. Km. 1 Carretera a Colón, Ajuchitlán, Colón, Querétaro, México. CP 76280. *Autor responsable y de correspondencia: Gómez-Rosales Sergio, Av. del Marqués 3100-40. Col. Centro Sur. Querétaro, Querétaro, México. CP 76090. gomez.sergio@inifap.gob.mx angeles.lourdes@inifap.gob.mx magueyjesus@gmail.com

\section{RESUMEN}

Las sustancias húmicas obtenidas de lombricompostas son una opción para mejorar la productividad y reducir las emisiones de amoniaco en las casetas de pollos de engorda. El objetivo del estudio fue evaluar la adición de lixiviado de lombricomposta crudo (LLC) o pasteurizado (LLP) al $20 \%$ en el agua de bebida sobre las variables productivas y de canal, la composición y ganancia de componentes químicos de pollos y camas y la retención y pérdidas de nitrógeno en pollos de 21 a 45 días de edad. Se ofreció una dieta convencional adicionada con antibióticos promotores del crecimiento durante todo el experimento. Los resultados indican que se mejoró $(P<0.05)$ el rendimiento de la pechuga en pollos adicionados con $20 \%$ de LLP en el agua de bebida comparados con los pollos que bebieron el LLC o solo agua. La adición de $20 \%$ de LLC o LLP no mejoró la retención de nutrientes en los pollos o las camas ni el balance o pérdidas de nitrógeno en pollos de 21 a 45 días de edad. EI LLP se puede a adicionar en el agua de bebida de pollos para mejorar el rendimiento de la pechuga.

Palabras Clave: pollos, lixiviado de lombricomposta, sustancias húmicas, nitrógeno, amoniaco.

\begin{abstract}
Humic substances obtained from vermicompost are an option to improve productivity and reduce ammonia emissions in broiler houses. The objective of the study was to evaluate the addition of $20 \%$ raw (RWL) or pasteurized (PWL) vermicompost leachate in the drinking water on production and carcass variables, broiler and litter chemical composition and gain, and nitrogen retention and losses in broilers from 21 to 45 days of age. A conventional diet supplemented with antibiotic growth promoters was offered throughout the experiment. Results indicate that breast performance was improved $(P<0.05)$ in broilers fed $20 \% \mathrm{PWL}$ in the drinking water compared to broilers that drank the RWL or water alone. The addition of $20 \% \mathrm{RWL}$ or PWL did not improve nutrient retention in chicks or litter or nitrogen balance or losses in 21- to 45-day-old chicks. PWL can be added to broiler drinking water to improve breast performance.
\end{abstract}

Keywords: broilers, wermicompost leachate, humic substances, nitrogen, ammonia. 


\section{INTRODUCCIÓN}

Las sustancias húmicas $(\mathrm{SH})$ se han evaluado desde hace algunos años como aditivos promotores del crecimiento y mejoradores de la salud en pollos de engorda y gallinas de postura (Sanmiguel et al., 2014; Arif et al., 2019). Los resultados de investigación en pollos de engorda adicionados con SH indican mejoras en el peso corporal, conversión alimenticia, peso de la canal y morfología de las vellosidades intestinales (Ozturk et al., 2012; Taklimi et al., 2012; Disethle et al., 2017). También se ha observado mayor digestibilidad y retención de energía, nitrógeno y cenizas en pollos suplementados con $\mathrm{SH}$ en el agua de bebida (Gomez-Rosales y Angeles, 2015).

Los componentes principales de las $\mathrm{SH}$ son los ácidos húmicos (AH), ácidos fúlvicos (HF) y huminas; se originan de la descomposición de la materia orgánica, son muy comunes en la naturaleza y se encuentran presentes de manera natural en el agua de bebida, suelo y lignito. Las SH son moléculas con estructura tridimensional que contienen un núcleo aromático con oxígeno y nitrógeno heterocíclicos; en las cadenas laterales hay grupos funcionales que confieren cualidades coloidales, espectrales, electroquímicas y de intercambio de iones (Lehmann y Kleber, 2015; Piccolo et al., 2019). Las SH se pueden encontrar en concentraciones de entre $8-12 \%$ en las compostas y lombricompostas preparadas con diferentes fuentes de materia orgánica y estiércol de animales domésticos (Gómez et al., 2013). Las SH se encuentran también, aunque en cantidades menores, en el líquido (lixiviado) que escurre de las camas de lombricompostas después de irrigarlas.

Dos de los mecanismos de acción propuestos sugieren que las $\mathrm{SH}$ actúan: 1) aumentando la permeabilidad de las membranas por su efecto detergente, ya que se comportan como surfactantes naturales y puede adsorberse en diferentes superficies que incluyen membranas biológicas aumentando la absorción de nutrientes (Gad El-Hak et al., 2012; Disetlhe et al., 2017) y 2) como agentes detoxificantes en el intestino debido a su poder reductor en la absorción de nitratos, fluorudos y metales pesados (Taklimi et al., 2012; Orsi, 2014). Las SH pueden inhibir la producción de amoniaco del suelo y rumen, lo que se ha asociado a una mayor eficiencia de síntesis de proteína microbiana (Zhang et al., 2013; Terry et al., 2018). También las SH reducen las emisiones de amoniaco del estiércol de cerdos suplementados con diferentes fuentes de $\mathrm{SH}$ por una probable reducción de la actividad ureásica bacteriana (Ji et al., 2006) y la concentración de amoniaco en excretas frescas de pollos suplementados con SH (Maguey-Gonzalez et al., 2018).

Los pollos de engorda son alimentados con dietas altas en proteína, lo que puede ocasionar excesos de amoniaco en el interior del intestino (Qaisrani et al., 2015; Lemme et al., 2019), causando daños en la mucosa como reducción de la altura de las vellosidades y profundidad de las criptas (Feng-Xiang et al., 2012; Zhang et al., 2015). En el interior de las casetas también se presentan mayores emisiones de amoniaco provenientes de las excretas de la cama, debido a la alta excreción de $\mathrm{N}$, provocando 
reducciones de la ganancia de peso e incremento de la conversión alimenticia (Zhang et al., 2015); además de daños en el flujo de moco, la acción ciliar y las membranas mucosas del tracto respiratorio (Wang et al., 2020; Zhou et al., 2020); reducción de los títulos de anticuerpos específicos y otras funciones inmunes, con incremento en la susceptibilidad a enfermedades e incremento en la mortalidad (Wei et al., 2015; Zhou et al., 2020). En un estudio previo se observó que pollos de engorda suplementados con un lixiviado de lombricomposta (LL) tuvieron mayor retención de $\mathrm{N}$ conforme se aumentó la dosis de LL, respecto al grupo control (Gomez-Rosales y Angeles, 2015); también se observó reducciones de hasta $30 \%$ en el contenido de amoniaco en excretas de pollos suplementados con SH extraídas de lombricomposta (Maguey-Gonzalez et al., 2018). La obtención de lombricompostas a partir de excretas animales representa una opción sustentable para el reciclaje de nutrientes y la mitigación de emisiones de gases de tóxicos como el amoniaco, y además son una fuente renovable de $\mathrm{SH}$ que adicionadas en los alimentos de pollos de engorda tienen la capacidad de mejorar el crecimiento, la retención de nitrógeno y reducir las emisiones de amoniaco de las excretas (MagueyGonzalez et al., 2018; Domínguez-Negrete et al., 2019). En trabajos previos en pollos suplementados con SH extraídas de lombricomposta los alimentos ofrecidos a los pollos estuvieron desprovistos de antibióticos promotores del crecimiento (Gomez-Rosales y Angeles, 2015; Maguey-Gonzalez et al., 2018; Domínguez-Negrete et al., 2019), pero se desconoce si los efectos positivos de las $\mathrm{SH}$ sobre el crecimiento y uso del nitrógeno en pollos suplementados con $\mathrm{SH}$ y antibióticos promotores del crecimiento en la misma dieta. Esta información es importante ya que en México la adición de antibióticos promotores del crecimiento en los alimentos es una práctica común. Con los antecedentes anteriores, se diseñó un estudio con el objetivo de evaluar la adición de lixiviado de lombricomposta crudo o pasteurizado sobre las variables productivas y de canal, composición y ganancia de componentes químicos de pollos y camas y la retención y pérdidas de nitrógeno en pollos de 21 a 45 días de edad.

\section{MATERIAL Y MÉTODOS}

\section{Ubicación, animales y diseño del experimento}

El estudio se llevó a cabo en el Centro Nacional de Investigación Disciplinaria en Fisiología y Mejoramiento Animal del Instituto Nacional de Investigaciones Forestales, Agrícolas y Pecuarias (INIFAP). El protocolo fue revisado y aprobado por el Comité de Ética de Uso de Animales y cumplió con la Norma Oficial Mexicana (NOM-062-ZOO, 1999). Se usaron 421 pollos Ross 308 de 21 a 45 días, alojados en piso (17-18 pollos/jaula), con ocho réplicas por tratamiento y se alimentaron con una dieta formulada con base en maíz y pasta de soya, para cubrir las recomendaciones de nutrientes de la estirpe, ofrecida a libre acceso. Se incluyó bacitracina metileno disalicilato (BMD) al $11 \%$ como antibiótico promotor del crecimiento a dosis de 500 ppm equivalentes a $55 \mathrm{ppm}$ de principio activo; y salinomicina al $12 \%$ a dosis de 500 ppm equivalentes a 60 ppm de 
principio activo para la prevención de coccidiosis. Los tratamientos fueron los siguientes: 1) los pollos recibieron agua de bebida directamente de una cisterna de almacenamiento conectada a bebederos de campana, 2) se mezcló $80 \%$ de agua de la cisterna con $20 \%$ de LL crudo (LLC) y 3) se mezcló $80 \%$ de agua de la cisterna con $20 \%$ de LL pasteurizado (LLP). EI LL se obtuvo de una lombricomposta preparada con estiércol de borrego. En el tratamiento 2, antes de mezclar el LL con el agua de bebida se filtró usando tela de algodón (pañalina). En el tratamiento 3 , el LL de filtró y se sometió a calentamiento a $65^{\circ} \mathrm{C}$ durante $1 \mathrm{~h}$ antes de mezclarlo con el agua. En los tratamientos 2 y 3 , la mezcla de agua y $L L$ se puso en garrafas de plástico de $20 \mathrm{~L}$ y se conectaron a los bebederos de campana. El contenido de AH, AF y ácidos húmicos totales (AHT) fue de 0.30, 0.33 y $0.66 \%$, respectivamente.

\section{Registro de variables productivas}

Se registró el peso corporal de los pollos al inicio y final del estudio y se estimó la ganancia diaria de peso (GDP, g/d) por la diferencia entre el peso final (42 d) y el peso inicial, dividido entre el número de días del experimento. También se llevó un control del alimento ofrecido y rechazado para estimar el consumo diario de alimento (CDA, g/d). La conversión alimenticia (CA) se calculó dividiendo el CDA entre la GDP. Se cuantificó el consumo diario de agua (CDAg, $\mathrm{ml} / \mathrm{d}$ ) y se calculó el consumo de agua/consumo de alimento. Se llevó registro diario de la mortalidad. Al final del experimento y después de un ayuno de $12 \mathrm{~h}$ se sacrificaron cinco pollos de cada corral y se registró el peso de la canal y la pechuga. El peso de la canal y la pechuga se expresaron en gramos (g) y el rendimiento se calculó en porcentaje (\%), dividiendo entre el peso corporal.

\section{Toma de muestras y análisis de laboratorio}

Al inicio del experimento, antes de poner los pollos en los corrales, se limpió el piso, colocando $11 \mathrm{~kg}$ de aserrín nuevo y se tomó una muestra; al final se pesó la cama completa y se tomó una muestra de cada corral. Al inicio del experimento se sacrificaron tres grupos de pollos de la misma parvada de los pollos usados en el experimento; al final se sacrificaron tres pollos por corral con un promedio de peso similar al peso promedio por corral. También se tomaron muestras del alimento cada semana.

Las muestras de camas fueron liofilizadas y molidas usando una criba de $2 \mathrm{~mm}$. Los pollos sacrificados fueron desplumados y se registró el peso de las plumas y del cuerpo, analizándolos por separado. El cuerpo completo fue molido en un molino para carne y se tomó una muestra representativa. El cuerpo y las plumas se liofilizaron por separado y se molieron. Las muestras de alimento fueron molidas usando una criba de $2 \mathrm{~mm}$. En las muestras de camas, cuerpo de los pollos, plumas y alimentos se determinó la materia seca (MS), cenizas $(C)$ y nitrógeno $(N)$. Todas las determinaciones de laboratorio se llevaron a cabo siguiendo las recomendaciones del AOAC (2019). 


\section{Cálculos de la composición química de los pollos y camas}

Para estimar la MS, $\mathrm{C}$ y $\mathrm{N}$ total de las camas y de los pollos al inicio y final del experimento en cada corral, se usaron las siguientes fórmulas:

$M S, C$ y $N$ en las camas, $k g=\%$ de $M S, C$ y $N \times$ peso de las camas;

MS, C y $\mathrm{N}$ en los pollos, $\mathrm{kg} *=(\%$ de $M S, C$ y $N$ de los pollos $\times$ peso de los pollos $)+$ (\% de MS, $C$ y $\mathrm{N}$ de las plumas $\times$ peso de las plumas, $\mathrm{kg}$ );

* La suma del $\mathrm{N}$ del cuerpo y las plumas se hizo en las cantidades equivalentes al peso del cuerpo y las plumas al inicio y al final del experimento.

Consumo total de alimento, $\mathrm{kg}=\mathrm{CDA} \times$ número de pollos por corral $\times 24$ días;

Consumo total de $M S, k g=\%$ de MS del alimento $\times$ consumo total de alimento;

Consumo total de $\mathrm{N}, \mathrm{kg}=$ Consumo total de $M S \times \%$ de $\mathrm{N}$ en el alimento;

\section{Cálculos de la ganancia de componentes químicos de los pollos y camas}

Para calcular la ganancia de componentes químicos en los pollos por corral, se usaron las siguientes fórmulas:

Ganancia de MS en los pollos, $\mathrm{kg}=$ MS total de los pollos al final MS total de los pollos al inicio del experimento;

Ganancia de $C$ en los pollos, $\mathrm{kg}=\mathrm{C}$ total de los pollos al final $C$ total de los pollos al inicio del experimento;

Ganancia de $N$ en los pollos, $\mathrm{kg}=\mathrm{N}$ total de los pollos al final $N$ total de los pollos al inicio del experimento;

Para calcular la ganancia de componentes químicos en las camas por corral se usaron las siguientes fórmulas:

Ganancia de MS en las camas, $\mathrm{kg}=$ MS total en la cama al final MS total en la cama al inicio del experimento;

Ganancia de $C$ en las camas, $\mathrm{kg}=C$ total en la cama al final $C$ total en la cama al inicio del experimento; 
Ganancia de $N$ en las camas, $\mathrm{kg}=\mathrm{N}$ total en la cama al final -

$N$ total en la cama al inicio del experimento;

\section{Cálculos de la retención y pérdidas de nitrógeno}

Para obtener la retención de $\mathrm{N}$ en $\mathrm{kg}$ y en porcentaje en los pollos y en las camas por corral, se usaron las siguientes fórmulas:

Retención de $N$ en pollos, $k g=N$ total en los pollos a los 45 días $N$ total en los pollos a los 21 días;

Retención de $N$ en los pollos, $\%=$

$\frac{N \text { total en los pollos a los } 45 \text { días }-N \text { total en los pollos a los } 21 \text { días }}{\text { Consumo Total de } N} \times 100$

Retención de $N$ en las camas, $\mathrm{kg}=N$ total en las camas a los 45 días $N$ total en las camas a los 21 días;

Retención de $N$ en las camas, $\%=$ $\frac{N \text { total en las camas a los } 45 \text { días }-N \text { total en las camas a los } 21 \text { días }}{\text { Consumo Total de } N} \times 100$

Retención de $N$ en pollos y camas, $\mathrm{kg}=\mathrm{N}$ total en los pollos y camas a los 45 días $N$ total en los pollos y camas a los 21 días;

Retención de $N$ en los pollos y camas, $\%=$ $N$ total en los pollos y camas a los 45 días $-N$ total en los pollos y camas a los 21 días Consumo Total de $N$ $\times 100$

La cantidad de $\mathrm{N}$ perdido en total, por pollo y por $\mathrm{kg}$ de pollo producido se calculó de la siguiente manera:

$N$ perdido en total, $\mathrm{kg}=$ Consumo total de $\mathrm{N}, \mathrm{kg}-$ retención de $\mathrm{N}$ en pollos y camas, $\mathrm{kg}$;

$N$ perdido por pollo producido, $g / d=\left(\frac{N \text { perdido en total, } \mathrm{kg}}{\text { Número de pollos }}\right) \div 24$;

$N$ perdido por pollo producido, $g / d=\left(\frac{N \text { perdido en total, } \mathrm{kg}}{\text { Total de kilos producidos }}\right) \div 24$; 


\section{Análisis estadístico}

Los resultados fueron sometidos a análisis de varianza bajo un diseño completamente al azar, usando los procedimientos de los Modelos Lineales Generales del paquete estadístico SAS. Previo a los análisis de varianza, se llevó a cabo la verificación de supuestos. En las variables GDP y CDA se transformaron usando el inverso multiplicativo y todas las variables expresadas en porcentaje se transformaron a arcoseno para cumplir con el supuesto de normalidad. Las diferencias estadísticas entre medias, se analizaron usando la metodología de la mínima diferencia significativa a $\mathrm{P}<0.05$.

\section{RESULTADOS Y DISCUSIÓN}

\section{Variables productivas y de la canal}

En la tabla 1 se presentan las variables productivas, peso y rendimiento de la canal y la pechuga. El peso inicial, peso final, consumo de alimento, ganancia de peso, conversión alimenticia, consumo de agua y el consumo de agua/consumo de alimento y mortalidad fueron similares entre los tres tratamientos evaluados. Estos hallazgos no concuerdan con los de un estudio en pollos adicionados con LLC en el agua de bebida (GomezRosales y Angeles, 2015), obteniéndose beneficios en el peso final, ganancia de peso y la conversión alimenticia; pero usando dietas desprovistas de antibiótico promotor del crecimiento y productos anticoccidiales. Uno de los propósitos del presente trabajo fue esclarecer si los efectos benéficos del LLC sobre el crecimiento de los pollos se podrían mantener a pesar de la presencia de BMD y salinomicina; sin embargo, los resultados de productividad muestran que los beneficios del LLC observados previamente usando dietas sin antibióticos se perdieron. La pasteurización del LL se llevó a cabo con la intención de eliminar los microorganismos presentes de manera natural y anulando algún posible efecto promotor del crecimiento que pudiera ejercer la flora benéfica; como se ha sugerido en pruebas de crecimiento de plantas adicionadas con LL, como fuente de SH (Canellas et al., 2015; Olivares et al., 2015). Otra diferencia importante fue que en el trabajo previo se usó un LL proveniente de una lombricomposta, hecha con estiércol de cerdo y borrego que contenía $0.47,0.14$ y $0.61 \%$ de AH, AF y AHT, respectivamente (Gomez-Rosales y Angeles, 2015); y en el presente trabajo se usó un LL obtenido de una lombricomposta, hecha con estiércol de borrego que contenía $0.30,0.33$ y $0.66 \%$ de $\mathrm{AH}$, AF y AHT, respectivamente.

El rendimiento de la pechuga fue mayor $(P<0.05)$ en los pollos que bebieron el LLP, respecto a los que bebieron solo agua y LLC (tabla 1). El peso y rendimiento de la canal y pechuga fueron similares entre tratamientos. En un estudio previo se obtuvo mayor rendimiento de la canal de pollos adicionados con SH extraídas de lombricomposta (Domínguez-Negrete et al., 2019). También se ha reportado mayor peso y rendimiento de la canal en pollos suplementados con cantidades crecientes de $\mathrm{SH}$ en el alimento y en el agua de bebida comparados con el grupo control no adicionado con SH (Ozturk et 
al., 2010; Ozturk et al., 2012). En un reporte previo se encontró que las SH sometidas a calentamiento por más de 40 min conservan sus propiedades detergentes, pero presentan una menor capacidad de transferencia de electrones de grupos químicos lábiles que se pierden durante el calentamiento (Visser, 1985); probablemente al reducirse las reacciones asociada al intercambio de electrones entre las $\mathrm{SH}$ y diferentes aceptores, y destacarse solo el efecto surfactante, aumentando la permeabilidad de las membranas; provocando el mayor rendimiento de pechuga con el LLP, comparado con el LLC.

Tabla 1. Variables productivas, peso y rendimiento de la canal y la pechuga

\begin{tabular}{|c|c|c|c|c|}
\hline & \multirow[b]{2}{*}{ Agua } & \multicolumn{2}{|c|}{ Lixiviado de lombricomposta } & \multirow{2}{*}{$\begin{array}{l}\text { Error estándar } \\
\text { de la media }\end{array}$} \\
\hline & & Crudo & Pasteurizado & \\
\hline \multicolumn{5}{|l|}{ Peso corporal } \\
\hline Día 21, kg & 0.66 & 0.64 & 0.66 & 0.011 \\
\hline Día 45, kg & 2.23 & 2.23 & 2.26 & 0.031 \\
\hline CDA, g/día & 142.48 & 141.92 & 142.87 & 1.922 \\
\hline GDP, g/día & 84.48 & 87.21 & 88.09 & 3.482 \\
\hline CDA/GDP & 1.69 & 1.65 & 1.64 & 0.056 \\
\hline CDAg, ml/día & 310.82 & 300.44 & 299.79 & 8.747 \\
\hline $\mathrm{CDAg} / \mathrm{CDA}$ & 2.19 & 2.11 & 2.08 & 0.065 \\
\hline Mortalidad, \% & 2.50 & 3.57 & 2.86 & 1.336 \\
\hline Pechuga, g & 488.61 & 488.12 & 497.49 & 11.360 \\
\hline Pechuga, \% & $23.55^{\mathrm{a}}$ & $23.43^{a}$ & $24.67^{b}$ & 0.397 \\
\hline Canal, g & 1193.31 & 1204.44 & 1205.26 & 23.301 \\
\hline Canal, \% & 57.58 & 57.89 & 59.82 & 0.751 \\
\hline
\end{tabular}

Variables productivas $n=8$. Pechuga y canal $n=40{ }^{a-b}$ Diferente letra en la misma fila muestra valores estadísticamente distintos $(P<0.05)$

\section{Composición y ganancia de componentes químicos de los pollos}

La composición química de los pollos sacrificados al inicio y final del experimento y la ganancia de componentes químicos se presentan en la tabla 2. La composición química inicial no se analizó estadísticamente porque solo se tomó una muestra representativa de los pollos. No hubo diferencias estadísticas en la composición química o ganancia de componentes químicos de los pollos al final. Los resultados no concuerdan con las mejoras en la eficiencia de uso de proteína y retención de $\mathrm{N}$ y $\mathrm{C}$ en pollos suplementados con SH (Gomez-Rosales y Angeles, 2015; Disetlhe et al., 2017), a través del método de colecta de excretas. 
Tabla 2. Composición química de los pollos al inicio y final del experimento y ganancia de componentes químicos

\begin{tabular}{|c|c|c|c|c|}
\hline & \multicolumn{3}{|c|}{ Lixiviado de lombricomposta } & \multirow{2}{*}{$\begin{array}{l}\text { Error estándar de la } \\
\text { media }\end{array}$} \\
\hline & Agua & Crudo & Pasteurizado & \\
\hline \multicolumn{5}{|c|}{ Composición química a los 21 días } \\
\hline Materia seca, \% & 26.79 & 26.79 & 26.79 & ND \\
\hline Cenizas, \% & 7.78 & 7.78 & 7.78 & ND \\
\hline Nitrógeno, \% & 12.06 & 12.06 & 12.06 & ND \\
\hline \multicolumn{5}{|c|}{ Composición química a los 45 días a } \\
\hline Materia seca, \% & 33.15 & 33.72 & 34.46 & 0.809 \\
\hline Cenizas, \% & 8.23 & 7.77 & 7.8 & 0.307 \\
\hline Nitrógeno, \% & 12.11 & 11.95 & 12.13 & 0.065 \\
\hline \multicolumn{5}{|c|}{ Ganancia de componentes químicos por corral a } \\
\hline Materia seca, $\mathrm{kg}$ & 9.74 & 9.78 & 10.55 & 0.352 \\
\hline Cenizas, kg & 2.31 & 2.09 & 2.19 & 0.127 \\
\hline Nitrógeno, kg & 1.18 & 1.16 & 1.22 & 0.048 \\
\hline
\end{tabular}

Composición química $n=24$. Ganancia de componentes químicos $n=8, N D=$ no determinado. a No se observaron diferencias estadísticas significativas entre tratamiento $(P>0.5)$

Se ha reportado que las $\mathrm{SH}$ son capaces de regular la disponibilidad de $\mathrm{N}$ para las plantas en el suelo, debido a sus propiedades adsorbentes, uniéndose directamente con el amoniaco o estimulando la actividad de bacterias desintegradoras que facilitan la absorción del $\mathrm{N}$ a través de las raíces (Canellas et al., 2015; Olivares et al., 2015). Se esperaba que este mismo efecto se pudiera llevar a cabo en el intestino de los pollos, reduciéndose los niveles de amoniaco liberado en el tracto digestivo, mejorando la salud, producción y retención de nutrientes (Qaisrani et al., 2015; Lemme et al., 2019). Probablemente la presencia de BMD y salinomicina contrarrestó los efectos de las SH observados en trabajos previos.

En la tabla 3 se muestra la composición química de las camas al inicio y final del experimento y la ganancia de los componentes químicos. Los componentes químicos al inicio no se analizaron estadísticamente porque solo se tomó una muestra representativa de las camas. No hubo diferencias estadísticas en la composición química o ganancia de componentes químicos de las camas al final. En estudios previos se ha reportado que las $\mathrm{SH}$ pueden inhibir la actividad ureásica presente en bacterias del suelo (Zhang et al., 2013) y las emisiones de amoniaco de excretas de cerdos y pollos suplementados con SH (Ji et al., 2006; Maguey-Gonzalez et al., 2018).

En el presente estudio se esperaba que las $\mathrm{SH}$ se unieran al amoniaco en el interior del intestino y en las camas, reduciendo las pérdidas de $N$ por volatilización, y en consecuencia, mayor retención de $\mathrm{N}$ en las camas al final. Probablemente la presencia de los antibióticos contrarrestó los efectos de las $\mathrm{SH}$ en las camas. 
Tabla 3. Composición química de las camas al inicio y final del experimento y ganancia de componentes químicos

\begin{tabular}{|c|c|c|c|c|}
\hline & \multicolumn{3}{|c|}{ Lixiviado de lombricomposta } & \multirow{2}{*}{$\begin{array}{l}\text { Error estándar de la } \\
\text { media }\end{array}$} \\
\hline & Agua & Crudo & Pasteurizado & \\
\hline \multicolumn{5}{|c|}{ Composición química a los 21 días } \\
\hline Materia seca, \% & 97.66 & 97.66 & 97.66 & ND \\
\hline Cenizas, \% & 4.77 & 4.77 & 4.77 & ND \\
\hline Nitrógeno, \% & 1.53 & 1.53 & 1.53 & ND \\
\hline \multicolumn{5}{|c|}{ Composición química a los 45 días } \\
\hline Materia seca, \% & 70.87 & 71.61 & 69.29 & 2.936 \\
\hline Cenizas, \% & 17.89 & 18.19 & 18.15 & 0.53 \\
\hline Nitrógeno, \% & 3.6 & 3.51 & 3.54 & 0.074 \\
\hline
\end{tabular}

Ganancia de componentes químicos por corral

$\begin{array}{lcccc}\text { Materia seca, } \mathrm{kg} & 10.46 & 9.4 & 9.6 & 1.199 \\ \text { Cenizas, } \mathrm{kg} & 3.26 & 3.16 & 3.17 & 0.213 \\ \text { Nitrógeno, kg } & 0.61 & 0.55 & 0.56 & 0.054\end{array}$

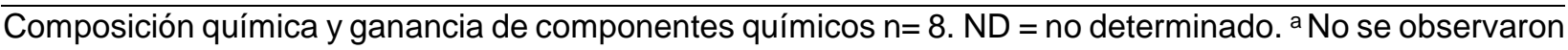
diferencias estadísticas significativas entre tratamiento $(P>0.5)$

\section{Retención y pérdidas de nitrógeno de los pollos y las camas}

En la tabla 4 aparecen los resultados de balance y pérdida de nitrógeno durante el experimento. Ninguna de las variables analizadas mostró diferencias entre tratamientos. Estos hallazgos no coinciden con el mayor rendimiento de la pechuga, que pudieran sugerir que hubo mayor eficiencia en el uso del $\mathrm{N}$ para la síntesis de proteínas musculares, ni con la mayor retención de $\mathrm{N}$ reportada en un trabajo previo en pollos suplementados con LLC (Gomez-Rosales y Angeles, 2015). Los resultados no concuerdan con las mejoras en la eficiencia de uso de proteína y retención de $\mathrm{N}$ y $\mathrm{C}$ en pollos suplementados con SH (Gomez-Rosales y Angeles, 2015; Disetlhe et al., 2017).

La falta de diferencias en el balance de $\mathrm{N}$, coincide con la falta de diferencias en la productividad de los pollos. La eficiencia de retención de $\mathrm{N}$ en los pollos, las camas y los pollos + camas fue de 41, 28 y $69 \%$, respectivamente. La retención de $\mathrm{N}$ en los pollos usando el método de sacrificio fue inferior a la retención de nitrógeno reportada (de 61$80 \%$ ) en pollos usados en estudios de balance de nitrógeno sometidos a alimentación restringida (Gómez et al., 2012; Gomez-Rosales y Angeles, 2015); pero es más acorde a la retención de nitrógeno (de $29-43 \%$ ) en pollos alimentados a libertad (Gomez y Angeles, 2011). 
Tabla 4. Retención y pérdidas de nitrógeno total

\begin{tabular}{|c|c|c|c|c|}
\hline & \multirow[b]{2}{*}{ Agua } & \multicolumn{2}{|c|}{ Lixiviado de lombricomposta } & \multirow{2}{*}{$\begin{array}{l}\text { Error } \\
\text { estándar de } \\
\text { la media }\end{array}$} \\
\hline & & Crudo & Pasteurizado & \\
\hline \multicolumn{5}{|l|}{ Balance de nitrógeno } \\
\hline Contenido de $\mathrm{N}$ de la dieta, $\%$ & 3.75 & 3.75 & 3.75 & ND \\
\hline Consumo diario de $\mathrm{N}, \mathrm{g}$ & 4.94 & 4.93 & 4.96 & 0.055 \\
\hline Consumo total de $\mathrm{N}, \mathrm{kg}$ & 2.07 & 2 & 2.08 & 0.095 \\
\hline Retención de $\mathrm{N}$ en los pollos, kg & 0.85 & 0.83 & 0.91 & 0.04 \\
\hline Retención en $\mathrm{N}$ los pollos/consumo de N, \% & 40.73 & 40.96 & 42.22 & 1.123 \\
\hline Retención de N en la cama, kg & 0.61 & 0.55 & 0.56 & 0.054 \\
\hline Retención de N en cama/consumo de N, \% & 29.23 & 27.09 & 27.26 & 1.646 \\
\hline Retención de $\mathrm{N}$ en pollos + cama, $\mathrm{kg}$ & 1.49 & 1.42 & 1.46 & 0.087 \\
\hline $\begin{array}{l}\text { Retención de } \mathrm{N} \text { en pollos y cama/consumo } \\
\text { de } \mathrm{N}, \%\end{array}$ & 69.96 & 67.84 & 68.93 & 2.52 \\
\hline \multicolumn{5}{|l|}{ Pérdidas de nitrógeno por corral y por pollo } \\
\hline $\mathrm{N}$ perdido total, $\mathrm{kg}$ & 0.58 & 0.61 & 0.6 & 0.118 \\
\hline $\mathrm{N}$ perdido total, $\%$ & 28.08 & 30.56 & 29.06 & 1.079 \\
\hline N perdido/pollo vivo/día, g & 1.37 & 1.48 & 1.41 & 0.133 \\
\hline $\mathrm{N}$ perdido/kg de pollo vivo/día, g & 0.62 & 0.68 & 0.63 & 0.058 \\
\hline
\end{tabular}

Balance de nitrógeno y pérdidas de nitrógeno $n=8$. ND = no determinado. ${ }^{a}$ No se observaron diferencias estadísticas significativas entre tratamiento $(P>0.5)$

En teoría, el resto del $\mathrm{N}$ no recuperado en los pollos fue excretado hacia la cama, esto es que, se esperaba recuperar el $59 \%$ del $\mathrm{N}$ excretado por los pollos; sin embargo, solamente se recuperó $28 \%$ de $\mathrm{N}$ en la cama, por lo que se asume que el $\mathrm{N}$ faltante se perdió en forma de amoniaco hacia el ambiente; esto es que $31 \%$ del $\mathrm{N}$ consumido y excretado se desprendió de la cama por volatilización en el interior de la caseta durante el periodo de 24 días, tiempo que los pollos permanecieron en el experimento. En las camas, las pérdidas de $\mathrm{N}$ en forma de amoniaco se deben a la mineralización microbiana de la urea y el ácido úrico que representan hasta el $80 \%$ del nitrógeno total excretado (Zhang et al., 2015).

Las pérdidas de $\mathrm{N}$ por pollo y por $\mathrm{kg}$ de pollo indican que se volatizó un promedio de 1.42 y $0.65 \mathrm{~g}$ de $\mathrm{N}$ al día, respectivamente; considerando que se requiere $0.216 \mathrm{~g}$ de $\mathrm{H}$ para la formación de $1 \mathrm{~g}$ de amoniaco a partir de $1 \mathrm{~g}$ de $\mathrm{N}$; estimando que por pollo y por kilo de pollo producido se generaron 1.72 y $0.78 \mathrm{~g}$ de amoniaco. La tasa de formación de amoniaco a partir de la cama depende de la temperatura y humedad ambiental principalmente, y la tasa de acumulación dentro de la caseta depende del tamaño de la caseta, número de pollos y grado de ventilación (Feng-Xiang et al., 2012; Wei et al., 2015). Sin embargo, se debe tomar en cuenta que cuando el amoniaco se libera de la cama, primero es inspirado por el pollo antes de distribuirse en el ambiente de la caseta. Si la caseta presenta una ventilación adecuada, los efectos perjudiciales del amoniaco 
en el pollo pueden ser leves; pero si la ventilación no es adecuada, el pollo permanecerá más tiempo expuesto a la toxicidad del gas, porque lo estará inspirando constantemente. Por lo anterior, es necesario seguir en la búsqueda de alternativas alimenticias y de manejo ambiental para mitigar las emisiones de gases contaminantes como el amoniaco en el interior de las casetas, para reducir sus impactos detrimentales en la productividad y salud de las aves.

\section{CONCLUSIONES}

Los resultados indican que se puede mejorar el rendimiento de la pechuga en pollos de engorda alimentados con dietas adicionadas con antibióticos promotores del crecimiento y adicionados con $20 \%$ de LLP en el agua de bebida. La adición de $20 \%$ de LLC o LLP no mejoró la retención de nutrientes en los pollos o las camas, ni el balance o pérdidas de $\mathrm{N}$ en pollos de 21 a 45 días de edad.

\section{LITERATURA CITADA}

ARIF M, Alagawany M, Abd El-Hack ME, Saeed M, Arain MA, Elnesr SS. 2019. Humic acid as a feed additive in poultry diets: A review. Iranian Journal of Veterinarian Research. 20(3):167-172. https://pubmed.ncbi.nlm.nih.gov/31656520/

ASSOCIATION of Official Analytical Chemists. 2019. Official methods of analysis of AOAC International. 21st Edition. Arlington, VA, USA. ISBN 0-935584-77-3. https://www.aoac.org/official-methods-of-analysis-21st-edition-2019/

CANELLAS LP, Olivares FL, Aguiar NO, Jones DL, Nebbioso A, Mazzei P, Piccolo A. 2015. Humic and fulvic acids as biostimulants in horticulture. Scientia Horticulturae. 196(11): 15-27. https://doi.org/10.1016/j.scienta.2015.09.013

DISETLHE ARP, Marume U, Mlambo V, Dinev I. 2017. Humic acid and enzymes in canola-based broiler diets: Effects on bone development, intestinal histomorphology and immune development. South African Journal of Animal Science. 47(6): 914-922. http://dx.doi.org/10.4314/sajas.v47i6.19

DOMÍNGUEZ-NEGRETE A, Gómez-Rosales S, Angeles ML, López-Hernández LH, Reisde Souza TC, López-García Y, Zavala FA, Tellez IG. 2019. Effect of the addition of humic substances as growth promoter in broiler chickens under two feeding regimens. Animals. 9(12): 1-15, e1101. http://doi:10.3390/ani9121101 
FENG-XIAN W, Bin X, Xiao-Fei H, Shao-Yu L, Fu-Zhu L, Quan-You S, Yu-Ping J, Lin-Yi W. 2012. The Effect of Ammonia and Humidity in Poultry Houses on Intestinal Morphology and Function of Broilers. Journal of Animal and Veterinary Advances. 11(19): 3641-3646. https://doi.org/10.1080/00071668.2020.1752912

GAD EL-HAK SH, Ahmed AM, Moustafa YMM. 2012. Effect of Foliar Application with Two Antioxidants and Humic Acid on Growth, Yield and Yield Components of Peas (Pisum sativum L.). Journal of Horticultural Science \& Ornamental Plants. 4(3): 318-328. https://doi.org/10.5829/idosi.jhsop.2012.4.3.262

GOMEZ S, Angeles ML. 2011. Effects of an enzymatically hydrolyzed yeast and yeast culture combined with flavomycin and monensin on finishing broiler chickens. International Journal of Poultry Science. 10(6): 433-439. https://doi.org/10.5713/ajas.2011.11316

GÓMEZ S, Angeles ML, Mojica MC, Jalukar S. 2012. Combination of an enzymatically hydrolyzed yeast and yeast culture with a direct-fed microbial in the feeds of broiler chickens. Asian-Australasian Journal of Animal Science. 25(5): 665 - 673. http://dx.doi.org/10.5713/ajas.2011.11316

GÓMEZ-ROSALES S, Angeles ML, Núñez-Hernández G, Figueroa-Viramontes U. 2013. Metodologías para la elaboración de compostas y lombricompostas de excretas de ganado de leche. Centro Nacional de Investigación Disciplinaria en Fisiología y Mejoramiento Animal, INIFAP-SAGARPA. Colón, Querétaro. INIFAP. 20: 1-52. ISBN: 978-607-37-0219-5.

https://pdfs.semanticscholar.org/581d/3131800723d95415a9a9b691ed672523ac41.pdf

GOMEZ-ROSALES S, Angeles ML. 2015. Addition of a worm leachate as source of humic substances in the drinking water of broiler chickens. Asia-Australasian Journal of Animal Science. 28(2): 215-222. http://dx.doi.org/10.5713/ajas.14.0321

JI F, McGlone JJ, Kim SW. 2006. Effects of dietary humic substances on pig growth performance, carcass characteristics, and ammonia emission. Journal of Animal Science. 84(9): 2482-2490. https://doi.org/10.2527/jas.2005-206

LEHMANN J, Kleber M. 2015. The contentious nature of soil organic matter. Nature. 528(11): 60-68. https://doi.org/10.1038/nature16069

LEMME A, Hiller P, Klahsen M, Taube V, Stegemann J, Simon I. 2019. Reduction of dietary protein in broiler diets not only reduces n-emissions but is also accompanied by several further benefits. Journal of Applied Poultry Research. 28(4): 867-880. https://doi.org/10.3382/japr/pfz045 
MAGUEY-GONZALEZ JA, Michel MA, Baxter MFA, Tellez G, Moore PA, Solis-Cruz B, Hernandez-Patlan D, Merino-Guzman R, Hernandez-Velasco X, Latorre JD, Hargis BM, Gomez-Rosales S, Tellez IG. 2018. Effect of humic acids on intestinal viscosity, leaky gut and ammonia excretion in a $24 \mathrm{hr}$ feed restriction model to induce intestinal permeability in broiler chickens. Animal Science Journal. 89(7): 1002-1010.

https://doi.org/10.1111/asj.13011|

OLIVARES FL, Aguiar NO, Rosa RCC, Canellas LP. 2015. Substrate biofortification in combination with foliar sprays of plant growth promoting bacteria and humic substances boosts production of organic tomatoes. Scientia Horticulturae. 183(1): 100-108. http://dx.doi.org/10.1016/j.scienta.2014.11.012

ORSI, M. 2014. Molecular dynamics simulation of humic substances. Chemical and Biological Technologies in Agriculture. 1(1): 1-14. https://doi.org/10.1186/s40538-0140010-4

OZTURK E, Ocak N, Turan A, Erener G, Altop A, Cankaya S. 2012. Performance, carcass, gastrointestinal tract and meat quality traits, and selected blood parameters of broilers fed diets supplemented with humic substances. Journal of the Science of Food and Agriculture. 92(1): 59-65. https://doi.org/10.1002/jsfa.4541|

OZTURK E, Ocak N, Coskun I, Turhan S, Erener G. 2010. Effects of humic substances supplementation provided through drinking water on performance, carcass traits and meat quality of broilers. Journal of Animal Physiology and Animal Nutrition. 94(1): 78-85. https://doi.org/10.1111/j.1439-0396.2008.00886.x|

PICCOLO A, Spaccini R, Savy D, Drosos M, Cozzolino V. 2019. The Soil Humeome: Chemical Structure, Functions and Technological Perspectives. In Sustainable Agrochemistry. S. Vaz Jr. (ed.) Springer Nature Switzerland AG. 187-222. https://doi.org/10.1007/978-3-030-17891-8_7

QAISRANI SN, Van Krimpen MM, Kwakkel RP, Verstegen MWA, Hendriks WH. 2015. Dietary factors affecting hindgut protein fermentation in broilers: A review. World's Poultry Science Journal. 71(1): 139-160. https://doi.org/10.1017/S0043933915000124

SANMIGUEL PRA, Aguirre PWJ, Rondón BIS. 2014. Perspectivas sobre el uso de sustancias húmicas en la producción aviar. Revista CES Medicina Veterinaria y Zootecnia. 9(1): 104-113. http://www.scielo.org.co/pdf/cmvz/v9n1/v9n1a10.pdf 
SECRETARÍA de Gobernación. [NOM-062-ZOO] Norma Oficial Mexicana. 1999. Especificaciones técnicas para la producción, cuidado y uso de los animales de laboratorio. Ciudad de México: Diario Oficial de la Federación. https://www.anmm.org.mx/bgmm/1864_2007/2002-138-3-295-298.pdf

TAKLIMI SM, Ghahri H, Isakan MA. 2012. Influence of different levels of humic acid and esterified glucomannan on growth performance and intestinal morphology of broiler chickens. Agricultural Science. 3(5): 663-668. https://doi.org/10.4236/as.2012.35080

TERRY SA, Ramos AFO, Holman DB, McAllister TA, Breves G, Chaves AV. 2018. Humic substances alter ammonia production and the microbial populations within a RUSITEC fed a mixed hay - Concentrate diet. Frontiers in Microbiology. 9(7): 1-19. https://doi.org/10.3389/fmicb.2018.01410

VISSER SA. 1985. Physiological action of humic substances on microbial cells. Soil Biology and Biochemistry. 17(4): 457-462. https://doi.org/10.1016/0038-0717(85)90009-4

WANG W, Shi Q, Wang S, Zhang H, Xu S. 2020. Ammonia regulates chicken tracheal cell necroptosis via the LncRNA-107053293/MiR-148a-3p/FAF1 axis. Journal of Hazardous Materials. $\quad 386(10): \quad 1-12, \quad$ e121626. https://doi.org/10.1016/j.jhazmat.2019.121626

WEI FX, Hu XF, Xu B, Zhang MH, Li SY, Sun QY, Lin P. 2015. Ammonia concentration and relative humidity in poultry houses affect the immune response of broilers. Genetics and Molecular Research. 14 (2): 3160-3169. https://doi.org/10.4238/2015.April.10.27

ZHANG WZ, Chen XQ, Zhou JM, Liu DH, Wang HY, Du CW. 2013. Influence of Humic Acid on Interaction of Ammonium and Potassium lons on Clay Minerals. Pedosphere. 23(4): 493-502. https://doi.org/10.1016/S1002-0160(13)60042-9

ZHANG J, Li C, Tang X, Lu Q, Sa R, Zhang H. 2015. Proteome changes in the small intestinal mucosa of broilers (Gallus gallus) induced by high concentrations of atmospheric ammonia. Proteome Science. 13(1): 1-14. https://doi.org/10.1186/s12953015-0067-4

ZHOU Y, Liu QX, Li XM, Ma DD, Xing S, Feng JH, Zhang MH. 2020. Effects of ammonia exposure on growth performance and cytokines in the serum, trachea, and ileum of broilers. Poultry Science. 99(5): 2485-2493. https://doi.org/10.1016/j.psj.2019.12.063 